\title{
School-based intervention that integrates nutrition education and supportive healthy school food environment among Malaysian primary school children: a study protocol
}

\author{
Choon Huey Teo ${ }^{1,2}$, Yit Siew Chin ${ }^{1,3^{*}}$ (D), Poh Ying Lim ${ }^{4}$, Shahril Azian Haji Masrom ${ }^{5}$ and Zalilah Mohd Shariff ${ }^{1}$
}

\begin{abstract}
Background: Malnutrition among school children may contribute to adverse health consequences such as noncommunicable diseases, poor cognitive performance, psychological distress and poor quality of life that may persist into adulthood. In order to prevent childhood malnutrition, an intervention programme that integrates nutrition education and healthy school food environment is needed to provide nutrition information and reinforce the skills on healthy eating behaviours in schools. This paper describes a study protocol of a school-based intervention programme that integrates nutrition education and healthy school food environment, namely School Nutrition Programme (SNP). The SNP is a primary prevention programme that promotes healthy lifestyle among primary school children in light of the high prevalence of malnutrition in Malaysian children.
\end{abstract}

Methods/design: This quasi-experimental study aimed to evaluate the effectiveness of the SNP between intervention and comparison groups before and after the SNP, and after a 3-month follow-up. The SNP consisted of two main components, whereby three nutrition education sessions were implemented by trained teachers using three standardised modules, and healthy school food environment was implemented by the canteen food handlers with the provision of healthy menu to children during school recess times. Children from intervention group participated in the SNP, in addition to the standard Physical and Health Curriculum. The comparison group attended only the standardised Physical and Health Curriculum and the school canteen food handlers were reminded to follow the standard canteen guidelines from the Ministry of Education Malaysia. The assessment parameters in evaluating the effectiveness of the programme were knowledge, attitude and practice on nutrition, eating behaviours, physical activity, body composition, psychological distress, cognitive performance and health-related quality of life. Assessments were conducted at three time points: pre-intervention, post-intervention and 3-month follow-up.

Discussion: It was hypothesised that the SNP would be effective in promoting healthy lifestyle among school children, and further contributes in preventing malnutrition problem, enhancing cognitive performance and improving healthrelated quality of life among school children. Findings of the present study can be expanded to other schools in future on ways to improve nutrition education and healthy school food environment.

(Continued on next page)

\footnotetext{
*Correspondence: chinys@upm.edu.my

'Department of Nutrition \& Dietetics, Faculty of Medicine \& Health Sciences,

Universiti Putra Malaysia, UPM, 43400 Serdang, Selangor, Malaysia

${ }^{3}$ Research Centre of Excellence, Nutrition and Non-Communicable Diseases,

Faculty of Medicine and Health Sciences, Universiti Putra Malaysia, UPM,

43400 Serdang, Selangor, Malaysia

Full list of author information is available at the end of the article
}

(c) The Author(s). 2019 Open Access This article is distributed under the terms of the Creative Commons Attribution 4.0 International License (http://creativecommons.org/licenses/by/4.0/), which permits unrestricted use, distribution, and reproduction in any medium, provided you give appropriate credit to the original author(s) and the source, provide a link to the Creative Commons license, and indicate if changes were made. The Creative Commons Public Domain Dedication waiver (http://creativecommons.org/publicdomain/zero/1.0/) applies to the data made available in this article, unless otherwise stated. 
(Continued from previous page)

Trial registration: UMIN Clinical Trial Registration UMIN000032914 (Date of registration: 7th June 2018, retrospectively registered).

Protocol version: 16th September 2019 \& Version 4

Keywords: Children, Malnutrition, School-based intervention, School nutrition, Nutrition education, School food environment

\section{Background}

The World Health Organization reported that 151 million and 51 million children under 5 years of age were stunted and wasted respectively; on the other hand, the number of overweight or obese children under the age of five was 38 million in 2017 globally [1]. More than half of stunted children, more than two thirds of wasted children and almost half of overweight children lived in Asia [2]. In South-East Asia, at least one in every four children under five was stunted; at least one in every 10 children under five was wasted and overweight respectively [2].

Overnutrition and undernutrition coexist among primary school children in Malaysia [3-6]. The National Health and Morbidity Survey (NHMS) 2017 showed that the prevalence of overnutrition (overweight $16.3 \%$; obesity $17.4 \%$ ) were two times higher than undernutrition (thinness 6.7\%; stunting 7.8\%) among Malaysian children aged 10 to 12 years [7]. In addition, the prevalence of overnutrition among primary school children (33.7\%) was higher than secondary school adolescents (28.5\%) [7]. Malnutrition refers to deficiencies, excesses or imbalances in a person's intake of energy and/or nutrients [8]. Undernutrition may increase risks of impaired physical and cognitive performance, as well as morbidity and mortality during childhood $[9,10]$. On the other hand, overnutrition is associated with increased risks of psychological distress such as depression, anxiety and social withdrawal [11-13]; low self-esteem, poor quality of life and cognitive deficits, especially in executive function $[14,15]$. In order to achieve the Sustainable Development Goals, it is important to recognise the role of prevention and health promotion in order to reduce the prevalence and the burden of diseases [16]. Therefore, designing an effective healthy lifestyle programme that is interconnected with the education programmes in primary school can improve health literacy that is needed to prevent this public health concern among primary school children.

In Malaysia, children aged 7 to 12 years old are generally enrolled in primary school levels for 6 years, which is known as Standards 1 to 6 [17]. Lifelong nutritional patterns are formed during childhood, which can reach at influential stages in their lives [18, 19]. Previous studies reported that Malaysian primary school children are at increased risk of poor dietary behaviours, including breakfast skipping, low fruits and vegetables intakes, unhealthy snacking behaviours and low physical activity [7, 20, 21], which may affect their nutritional status and expose children to malnutrition [22, 23], lower cognitive performance and poor quality of life [24-26]. There is a need to have a holistic nutrition intervention programme that promotes healthy eating and active living for all primary school children, so that they can have a healthy lifestyle, good nutritional status, better cognitive performance and good quality of life.

This paper describes the protocol of School Nutrition Programme (SNP) that consisted of two main components, namely nutrition education and healthy school food environment. The present study aimed to evaluate the effectiveness of SNP on differences in primary outcome measures including anthropometric measurements, knowledge, attitude and practice on nutrition, eating behaviours, physical activity; and secondary outcome measures including psychological distress, cognitive performance and health-related quality of life among primary school children. A process evaluation was focused on the attendance rate of children during the School Nutrition Campaign, teachers and canteen food handlers during training of trainers; and the programme feedbacks from children, teachers and canteen food handlers after the study.

\section{Methods/design Study design}

The present study design was a quasi-experimental design. Out of 147 National Primary Schools in Batu Pahat district, a total of 143 National Schools met the inclusion criteria of being co-educational, non-religious, nonspecial educational, multi-ethnic and government funded schools. Then, these 143 schools were stratified based on the three main school types, namely i) National Primary School (SK), ii) National Chinese Primary School (SJKC) and iii) National Tamil Primary School (SJKT). Two schools from each stratified group were selected. One school was selected as Intervention Group (IG) and one as Comparison Group (CG) from each school type. All children from these selected schools were invited to participate in the study. 


\section{Participants}

This study was conducted in six selected schools in Batu Pahat District, Johor State, Malaysia. School authorities (principal and teachers) and canteen food handlers from three selected intervention schools were invited to participate in the study. All Malaysian children (males and females) who were enrolled in Standards 1 to 5 (aged 7 to 11 years) and who was either Malay, Chinese or Indian were invited in the study (Fig. 1). Upon request from the Ministry of Education (MOE) Malaysia, children in Standard 6, who would be attending the National Primary School Evaluation Test, were excluded from the study. In addition, children with learning disorders, obvious genetic disorders such as attention deficit hyperactivity disorder (ADHD) were excluded from the study. The children were assessed prior to the start of the programme (Pre-Intervention), one-week right after completing SNP (Post-Intervention I) and three-month follow-up without intervention (Post-Intervention II).

\section{Sample size calculation}

The sample size for primary school children was calculated using Aday's (2006) equation [27]. Based on a previous study in Malaysia, the mean difference of waist circumference between intervention and comparison group after the intervention programme was taken into consideration to calculate the sample size of the SNP [28].

$$
\mathrm{n}=\frac{2 \sigma^{2}\left[\mathrm{Z}_{1-\sigma / 2}+\mathrm{Z}_{1-\beta}\right]^{2}}{\left(\mu_{1}-\mu_{2}\right)^{2}}
$$

Based on the study, the mean change in waist circumference for intervention group after the intervention programme was $0.1 \mathrm{~cm}$, whereas mean change in waist circumference for comparison group after the intervention programme was $2.2 \mathrm{~cm}$. The calculated sample size was 195 children in intervention and comparison groups, respectively. The sample size was also adjusted for a response rate of $80.0 \%$. The intended sample size was 488 primary school children from Standards 1 to 5 in Batu Pahat District. Considering a power of $80 \%$ and a level of significance of $5 \%$, a total of 244 children was needed for each intervention and comparison group, respectively. The small effect size of 0.09 was obtained.

\section{Description of School Nutrition Programme intervention}

The SNP aimed to prevent malnutrition among primary school children. The rationale of SNP was to increase

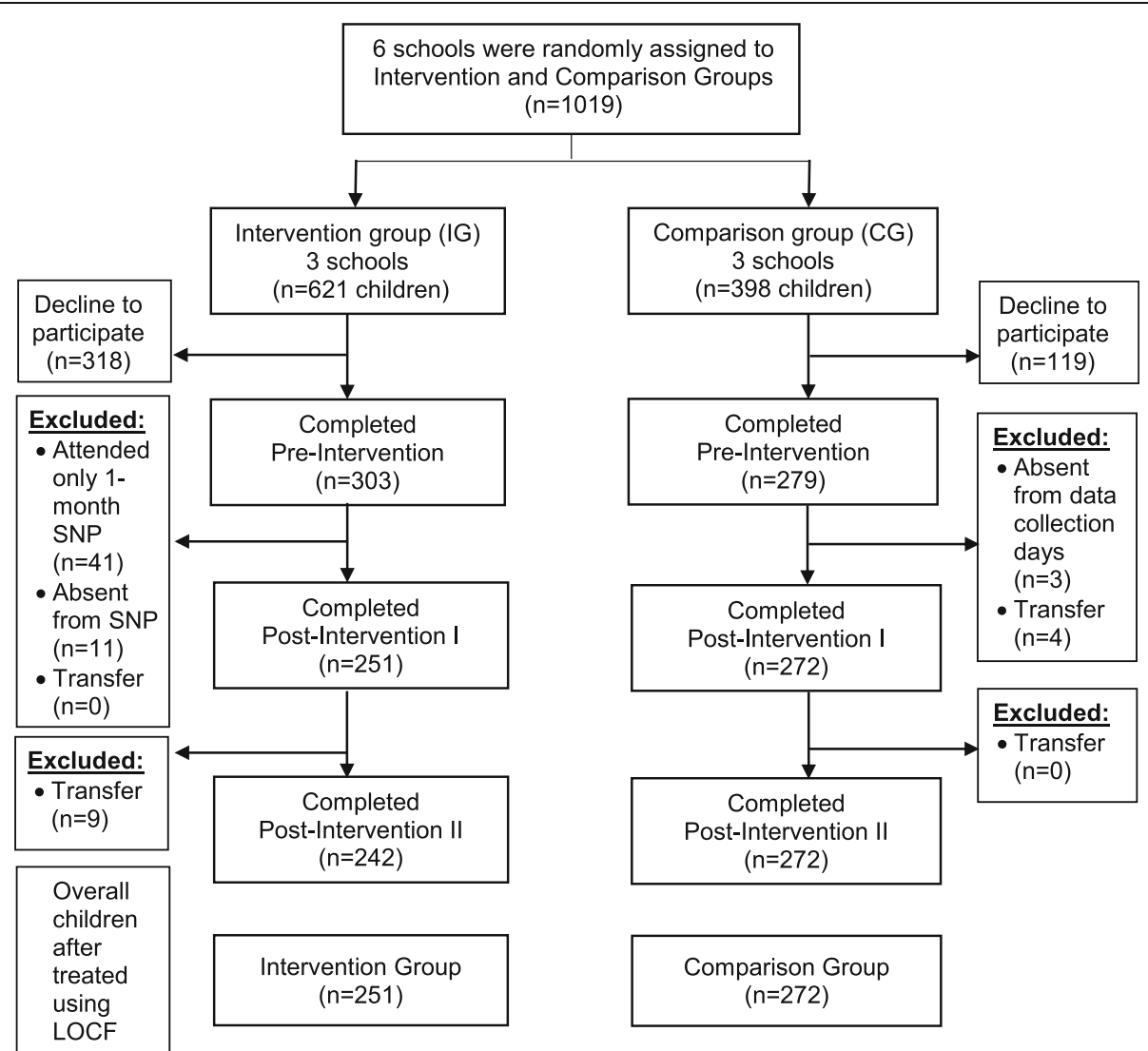

Fig. 1 Children's flow diagram 
the children's nutrition and physical activity knowledge, attitude and practices to optimise their healthy lifestyle by encouraging them to eat healthily and stay physically active. The SNP is based on the Social Cognitive Theory (SCT) [29], which emphasises the importance of social and environmental factors in determining the psychosocial and behavioural risk factors of both undernutrition and overnutrition. The SNP is composed of two main components: (1) delivering nutrition education through School Nutrition Campaign for 3 months; and (2) serving healthy menu during school recess time to the children over a period of 3 months. The children of the intervention group participated in three School Nutrition Campaigns, in addition to the standard Physical dan Health Curriculum. The children in the comparison group received standard Physical and Health Curriculum and school canteen food handler were reminded to follow canteen guidelines by MOE Malaysia.

For nutrition education component, the researchers conducted three School Nutrition Campaigns using the three different nutrition education modules and education materials of Healthy Kids Programme [30]. These nutrition education modules included four main aspects, namely health awareness, nutrition, physical activity and hygiene. Specifically, module 1 comprised six topics with the goal of educating primary school children on the importance of healthy lifestyle and introduce the concept of food pyramid, physical activity and personal hygiene. Module 2 comprised six topics with the goal to provide more in-depth knowledge on food groups in the Malaysian Food Pyramid and Physical Activity Pyramid. Module 3 comprised five topics with the goal to strengthen the important skills to apply the knowledge that the children learnt from module 1 and module 2. These modules incorporated the "fun while learning" concept, with various attractive engagement activities such as act and guess, role-play, quiz and detective games. There was a fourweek gap between each campaign, with at least 6 hours per campaign.

The training of trainers (TOT) aimed to provide hands-on training to the teachers to carry out the nutrition education component of the SNP, and to empower them to convey healthy lifestyle messages to the children during the School Nutrition Campaigns. As there were three modules, three separate sessions of the TOTs (1 day training session for each TOT) were needed to be completed by the teachers. The trained teachers conducted the nutrition education sessions to the children during the School Nutrition Campaigns and each topic took approximately 1 hour to deliver.

The second component of the SNP is a supportive healthy school food environment, which aimed to increase the availability of healthy foods in primary school canteen and to provide training to the canteen food handlers. A 1-day TOT of canteen food handlers was conducted to all food handlers by using a revised Healthy Catering Module from the Nutrition Division, $\mathrm{MOH}$ Malaysia [31], with incorporation of a few interactive games during the training. The food handlers learned to serve healthier foods, the fundamentals of preparing healthy foods, recipe modification, menu planning and healthy cooking techniques. All canteen food handlers needed to fulfil the basic requirements from the MOE Malaysia, which were to attend the Food Handling Course and compulsory get anti-typhoid vaccination [32].

During the TOT of canteen food handlers, the canteen food handlers discussed and planned a one-month healthy menu together with the researchers. One-week after the TOT of canteen food handlers, all canteen food handlers prepared four menus assigned by the researchers during Menu Testing Day. Stakeholders (such as principal, student affair teacher, Parent Teacher Association and school children) were invited to taste the healthy menu. Their comments and feedbacks were noted and used to further improve the menu. The healthy menu should contain a variety of food groups, such as from rice, noodle or bread, vegetable, fruit, and meat or chicken. Local food availability and children's acceptability were taken into consideration when planning for the healthy menu. All menus were monitored by researchers and the trained teachers during the intervention period. Therefore, students would be able to practise healthy eating during school recess. Throughout the study period, the researchers would inform and remind the children about the importance of programme adherence.

\section{Measurements}

All participants in both intervention and comparison groups were assessed prior to the start of the programme (Pre-Intervention), one-week right after completing the School Nutrition Campaigns (Post-Intervention I) and three-month afterwards without intervention (Post-Intervention II). The questionnaires were prepared in Malay, Chinese and Tamil languages to ease the children in understanding the questionnaires better. The anthropometric measurements and cognitive assessments of the children were conducted by trained researchers.

\section{Socio-demographic background}

In the present study, socio-demographic background of children in both intervention and comparison groups were determined. Children answered the items regarding their socio-demographic background, such as sex, ethnicity, age and date of birth, while parents answered questionnaires regarding parental education level and monthly household income. 


\section{Impact evaluation}

Impact evaluation included primary outcome measures, namely anthropometric measurements, knowledge, attitude and practice on nutrition, eating behaviours, physical activity; and secondary outcome measures included psychological distress, cognitive performance and health-related quality of life.

\section{Primary outcomes}

\section{Anthropometric measurements}

Body weight of the children was measured using weighing scale and recorded to the nearest $0.1 \mathrm{~kg}$. Height of the children was measured using stadiometer and recorded to the nearest $0.1 \mathrm{~cm}$. Body weight and height were used for BMI calculation using the BMI formula $\mathrm{BMI}=$ weight $(\mathrm{kg}) /$ height $^{2}\left(\mathrm{~m}^{2}\right)$ [33]. The $\mathrm{z}$-scores of weight-for-age (WAZ), height-for-age (HAZ) and BMIfor-age (BAZ) were computed using WHO AnthroPlus software [34], the nutritional status of children was obtained by comparing the $\mathrm{z}$-scores against the WHO Growth Reference 2007 tables [33]. Waist circumference was measured by using Lufkin Executive diameter steel tape Model W606 PM. Abdominal obesity was determined by referring to the 90th percentile cut-off-point of waist circumference for children aged 7-11 years [3]. Percentage of body fat for children aged 10-11 years was measured using Omron HBF-306 Body Fat Analyzer Scale (Omron Corporation, Japan). Based on the obtained body fat percentage, the children were categorised into normal and high body fat percentage [35]. All assessments by qualified researchers followed the International Society for the Advancement of Kinanthropometry (ISAK) procedures.

\section{Knowledge, attitude and practices on nutrition}

Knowledge, attitude and practices on nutrition of children were measured by using the knowledge, attitude and practices on the modules of Healthy Kids Programme [30]. The questionnaire consisted 30 knowledge items in the format of multiple choices. Each knowledge on nutrition item had four answer options. Each correct response was allocated one point and an incorrect answer was allocated 0 point. The possible range of scores for knowledge was between 0 and 30. A higher score indicated a higher level of knowledge on nutrition. Besides, there were 30 attitude items. Each attitude item was measured on a 3-point Likert scale; from agree (3 points) to disagree (1 point). The possible range of scores for attitude was between 10 and 30. The total attitude score was summed up and a higher attitude score indicated better attitude on nutrition. In addition, there were a total of 30 practice items. The practice items were assessed on a 5 point-frequency scale, ranging from almost every day [5] to never [1]. The possible range of scores for practice was 10-50. All the frequency points were summed up, and a higher score indicated healthier practice on nutrition by the children.

\section{Eating Behaviours}

Eating behaviours of the children, which included main meal consumption and snacking behaviour were assessed based on the Eating Behaviours Questionnaire (EBQ) [36]. This questionnaire was designed to measure the frequency of main meal consumption (breakfast, lunch and dinner) and frequency of snacking between meals (morning tea break, evening tea break and supper). The frequency of meal consumption was based on number of days consumed in a week [36]. For snacking behaviour among the children, the number of days that the children consumed morning tea, afternoon tea and supper in a week were asked.

\section{Physical activity}

Physical Activity Questionnaire for Children (PAQ-C) is appropriate for elementary school-aged children (approximately ages 8-14 years) who are currently in the school system and have recess as a regular part of their school week $[37,38]$. The PAQ-C is a self-administered, 7-day recall instrument, which provides a summary physical activity score derived from nine items, each scored on a 5-point scale. Item 1 (spare time activity) from no activity $=1 ; 7$ times or more $=5$. Items 2 to 8 (PE, recess, lunch, right after school, evening, weekends) from lowest activity response $=1$ or highest activity response $=5$. Item 9 (mean of all days of the week) from none $=1$; very often $=5$. Item 10 (identifies students who are unusual active during the previous week). By adding up all means of the first nine items in PAQ-C, a summative score of physical activity was obtained. A score of 1 indicated low physical activity level, whereas a score of 5 indicated high physical activity level. The Cronbach's alpha of PAQ-C was 0.89 and it was higher than the acceptance level (0.60) [37].

\section{Secondary outcomes Psychological distress}

The Revised Child Anxiety and Depression Scale (RCADS30) was used to measure symptoms of psychological distress, namely separation anxiety disorder, social phobia, generalised anxiety disorder, panic disorder, obsessive compulsive disorder, and major depressive disorder [39]. A 30-item version of RCADS (RCADS-30) was developed whereby this briefer version has five to six items per subscale. A self-report scale was used with subscale corresponding to separation anxiety disorder (5 items), social phobia (5 items), generalised anxiety disorder (5 items), panic disorder (5 items), obsessive compulsive disorder (5 items) and major depressive disorder (5 items). Items were scored 0-3 corresponding to never, sometimes, often and 
always respectively. A higher score indicated having more severe anxiety and depression symptoms [40]. The internal consistency of the RCADS-30 was tested and the Cronbach's alpha for total scale was 0.89 , with alpha coefficients for the separate RCADS-30 subscales ranging from 0.68 (obsessive-compulsive disorder) to 0.78 (generalised anxiety disorder) [40].

\section{Cognitive performance}

Raven's Coloured Progressive Matrices (CPM) was used to assess cognitive performance of children [41]. This test contained 36 items with three sets of 12 items in each (sets $\mathrm{A}, \mathrm{AB}$ and $\mathrm{B}$ ) from the standard matrices. Most items were presented on a coloured background to make the test visually stimulating for participants. Each child needed around $15 \mathrm{~min}$ to complete the test. Children were asked to find and complete the missing pattern. Each correct answer was given 1 mark. The raw score on the coloured progressive matrices test ranged between 0 and 3 . The child's mental age is thus the age at which the median score is equal to his or her raw score. This score was converted into a standard score based on the Raven's CPM norm table. A higher score indicated better cognitive performance.

\section{Health-related quality of life}

Pediatric Quality of Life Inventory 4.0 (PedsQL 4.0) was used to assess health-related quality of life (HRQoL) among children aged 8-12 years [42]. There was a total of 23 items from 4 subscales for child report: My health and activities; My feelings, I can get along with others and About school. Children were asked to recall healthrelated problems in the past 1 month and rate each item on a five-point Likert scale $(0=$ never, $1=$ almost never, $2=$ sometimes, 3 = often, $4=$ almost always). Scores of all items were plotted on a $0-100$ point scale $(0=100,1=$ $75,2=50,3=25,4=0)$. Higher total PedsQL scores corresponded to better HRQoL. The Cronbach's alpha of PedsQL was 0.89 and it was higher than the acceptance level (0.60) [43].

\section{Process evaluation}

Process evaluation of the SNP consisted of evaluation of attendance rate and programme feedback among the intervention groups. The attendance rate was assessed by using the attendance list for implementation of School Nutrition Campaigns 1, 2 and 3, respectively. Teachers needed to use the attendance list prepared by the researchers and took the attendance of children for each camp. Overall attendance rate and attendance by each camp were calculated. Programme feedback forms for three camps were self-administered by children after completing each of the campaigns. Percentage of programme feedback forms submitted by each camp was calculated. The attendance rate and programme feedback of TOT for teachers and TOT for canteen food handlers were also assessed in this study.

\section{Data analysis}

The evaluation of the study was based on the modified intention-to-treat analysis to retain children in the study [44]. Children who fulfilled the following criteria would be included in the data analysis process:

i. Children of the intervention group who have attended at least two camps in the School Nutrition Programme

ii. Children from both the intervention and comparison groups who had completed PreIntervention, Post-Intervention I and PostIntervention II.

All statistical analysis will be conducted using IBM SPSS statistics 24.0. Descriptive analysis such as frequency and percentages, will be reported for categorical variables, while means and 95\% confident interval will be reported for continuous variables. Skewness test of normality will be used to assess the normality of continuous variables. A value of skewness within the range of \pm 2.0 will be considered as normally distributed [45]. The Chi-square Test or Fisher's Exact Test will be used to determine the association between two or more groups of categorical variables. The Independent sample t-test will be used to compare the mean values of the continuous variables between intervention and comparison groups and Paired sample t-test will be used to compare the mean values at pre-intervention and postintervention within the intervention and comparison groups.

Simple linear mixed model will be conducted to determine the association of each covariate (such as time, group, child's age, parental education level and school type) with all the dependent variables (namely knowledge, attitude and practice on nutrition, eating behaviour, physical activity, anthropometric measurement, psychological distress, cognitive performance and health-related quality of life). Multiple linear mixed model will be conducted to investigate the dependent variables between groups (intervention and comparison) over time, adjusted for covariates. Interaction term of time and group will be included in the model. The differences in all continuous variables between intervention and comparison groups will be determined at three time points (Pre-Intervention, Post-Intervention I and PostIntervention II). The statistical significance will be set at $p<0.05$. The data will be stored for future use after the completion of the study. 


\section{Ethical approval}

Ethical approval for this study was obtained from the National Medical Research Registry (NMRR) and Medical Research Ethics Committee (MREC) of Ministry of Health $(\mathrm{MOH})$, Malaysia (NMRR-17-1273-36,187 IIR). Permissions for field data collection in primary schools were obtained from MOE Malaysia, State Department of Education of Johor, District Education Centre of Batu Pahat and selected schools before conducting the study. The researchers explained the study protocol to the children and their parents by using the information sheet. Prior to data collection, written consents were obtained from children, their parents/guardians, school authorities (principal and teachers), and canteen food handlers, respectively.

\section{Discussion}

The present study will evaluate the effectiveness of a school-based intervention to prevent malnutrition among Malaysian primary school children. The threemonth SNP that integrated nutrition education and healthy school food environment aimed to improve knowledge, attitude and practice on nutrition, eating behaviours, physical activity, cognitive performance and health-related quality of life; and to reduce BMI-for-age among primary school children. In order to achieve the primary outcomes of the present study, Koo and colleagues (2018) showed that a 12-week nutrition education intervention was enough to significantly increase practice of wholegrain foods consumption on a daily basis, lower body mass index-for-age z-score, body fat percentage and waist circumference among the intervention group, which was in line with previous study [46].

In Malaysia, several nutrition intervention programmes have been carried out among children aged 8 years [ 47 , 48], adolescents [49] and children with overweight and obesity problem [50], which reported effectiveness of nutrition education intervention programmes in improving knowledge, attitude and practices on nutrition. The efforts were mainly for nutrition education promotion on healthy eating and physical activity. For instance, a 3-week Nutrition Education Programme among 8-year-old children in Malaysia aimed to improve nutrition knowledge, attitude, and practice found that knowledge and attitude had improved at post-intervention and 6-months follow-up, but practice score did not increase throughout the study in the intervention group over time [47]. Another Nutrition Education Programme by Zalilah et al. [28] focused among 8-year-old children showed that there were positive impacts on nutrition knowledge, attitude and practice after the intervention, but no follow-up result was reported after the programme. Zalilah et al. suggested that healthy family environment, healthy food availability and accessibility in schools should be included in nutrition intervention in order to sustain nutrition practices [48].
Tee et al. [31] also recommended that a successful nutrition intervention should include content and teaching strategies that are developmentally appropriate for the children and address changes in the environment. To date, there is no published study that focuses on promoting healthy school food environment in Malaysia. Therefore, it is important to develop an intervention that integrates nutrition education and school food environment, especially the school canteen that can exert a strong influence on children's food decisions.

In Malaysia, primary school children consume their morning tea break during recess at school canteens, some children would take their lunch at school when they have tutorial classes or co-curricular activities. The Healthy School Canteen Guideline was developed and implemented by MOE Malaysia since year 2011. These guidelines aimed to improve the food service of school canteens and cultivate a balanced and healthy food intake in schools [32]. Based on the assessments of 113 primary school canteens in Batu Pahat district conducted in the year 2015, about one-third (33.6\%) of the canteens were under the "Dissatisfactory" category, indicating non-compliance to the guidelines [51]. Most of the canteen caterers sold cordial drinks, carbonated drinks and fried foods especially processed foods like nugget, burger and hotdog; while insufficient green vegetables and fruits were prepared for the students [51]. Frederike et al. reported that the nutritional intake among United States school children was significantly affected by the school food environment because children spend many hours at school each day [52, 53]. A systematic review and meta-analysis stated the effectiveness of healthy school food environment in increasing the consumption of fruits and vegetables, while reducing total fat intake, saturated fat intake and sodium intake [54]. In order to increase healthy food availability in primary school canteens, it is important to address the concerns and knowledge of canteen food handlers [55]. However, Lessa et al. found that food handlers had a significantly lower food nutrition knowledge score than the general public [56]. Therefore, an integrate intervention, which includes collaboration between canteen food handlers and nutritionist is needed to ensure that appealing, healthy menu is prepared for the primary school children.

In Japan, besides serving nutritious food, the school lunch programme namely Shokuiku also serves as an important nutrition educational approach for school children to acquire proper nutrition knowledge, and to impart a sense of gratitude in children [57]. To the best of our knowledge, the present study is the first school-based nutrition intervention that integrates nutrition education and healthy school food environment among primary school children in Malaysia. This School Nutrition Programme is 
hypothesised to improve knowledge, attitude and practices on nutrition, eating behaviours, physical activity, anthropometric assessments, psychological distress, cognitive performance and health-related quality of life among Malaysia primary school children. Therefore, the examination on the effectiveness of a successful school nutrition approach within school setting is of great importance.

The sustainability of the intervention beyond the study duration will be considered through training of school teachers on the nutrition education curriculum and the transformation of school canteens by preparing a variety of nutritious menu for children during school recess. The World Health Organization reported that implementation of a comprehensive programme that promotes healthy school food environment, health and nutrition education and physical activity among school-age children and adolescents can end childhood malnutrition issues [1, 28]. Establishment of standards for meals provided in schools by increasing the availability of whole grains foods sold in schools that meet healthy nutrition guidelines and eliminate the provision or sales of unhealthy foods, such as sugar sweetened beverages and energy-dense, nutrient-poor foods, in the school environment are important. Previous local studies suggested that modifiable dietary habit strategies such as increasing fruits and vegetables intake, increasing whole grains intake, limiting consumption of sweetened drinks and controlling portion sizes were needed to combat childhood obesity $[58,59]$. Importance of proper diets has been recognised not only for the control of lifestyle-related diseases, but more so in various physical aspects to improve the quality of life, including good growth of children [60].

Findings of the current study can be used by future researchers, health professionals, policy makers and school authorities to plan and implement the intervention in preventing malnutrition among primary school children in Malaysia. Furthermore, policy makers (including Parent Teacher Association), health agencies, programme planners and community leaders can use the data obtained from this study for planning and implementing effective policies as well as other intervention programmes that consist of nutrition education and healthy school food environment for Malaysian children to promote healthy lifestyle. The current study is important as it may raise awareness of the public, especially school teachers and canteen food handlers to give more attention towards the importance of nutrition education and healthy menu in canteens to Malaysian primary school children. This intervention study is in line with the National Plan of Action for Nutrition of Malaysia [NPANM] III (2016-2025), where nutrition education promotion is needed in schools and School Meal Programme has to be implemented to at least three schools by the year 2020 and at least six schools by the year 2025 in every state in Malaysia [61].

\section{Abbreviations}

BMI: Body Mass Index; ISAK: International Society for the Advancement of Kinanthropometry; MOE: Ministry of Education; $\mathrm{MOH}$ : Ministry of Health; NHMS: National Health and Morbidity Survey; PTA: Parent Teacher Association; SNP: School Nutrition Programme; WHO: World Health Organization

\begin{abstract}
Acknowledgements
We thank the Nutrition Society of Malaysia for granting us permission to use the modules and education materials of the Healthy Kids Programme, which were developed with the support of an unconditional educational grant from Nestle Malaysia. We also sincerely thank the schools, teachers and students who participated in this study and are extremely thankful for the support and cooperation given from the Department of Health and Education of Batu Pahat District and the Malaysian Ministry of Education.
\end{abstract}

\section{Authors' contributions}

TCH and CYS conceived and designed the study. LPY, SAHM and ZMS contributed substantially to the design and testing of the intervention. TCH and LPY developed the statistical approaches. TCH and CYS wrote the manuscript. LPY, SAHM and ZMS contributed to drafting of the manuscript and provided critical input. All authors have read and approved the final manuscript.

\section{Funding}

The present study is not considered as being externally funded. The Nutrition Society of Malaysia funded the printing of modules and education materials of the Healthy Kids Programme, which were used in the nutrition education component of the SNP. The Nutrition Society of Malaysia did not fund the study protocol and implementation, except for the permission to use the modules and education materials of the Healthy Kids Programme in this intervention study.

\section{Availability of data and materials}

Data sharing is not applicable to this article as no datasets were generated or analysed during the current study.

\section{Ethics approval and consent to participate}

Ethical approval for this study was obtained from the National Medical Research Register (NMRR) and Medical Research Ethics Committee (MREC) of $\mathrm{MOH}$, Malaysia (NMRR-17-1273-36,187 IR). Permission for field data collection in primary schools was obtained from MOE Malaysia, State Department of Education of Johor and District Education Centre of Batu Pahat before the study. Written consents were obtained from children, their parents/ guardians, school authorities (principal and teachers), and canteen food handlers, respectively.

Consent for publication

Not applicable.

\section{Competing interests}

At the time of submission, CYS served as an Associate Editor of BMC Public Health.

\section{Author details}

${ }^{1}$ Department of Nutrition \& Dietetics, Faculty of Medicine \& Health Sciences, Universiti Putra Malaysia, UPM, 43400 Serdang, Selangor, Malaysia.

${ }^{2}$ Department of Nutrition, Batu Pahat District Health Office, 83000 Batu Pahat, Johor, Malaysia. ${ }^{3}$ Research Centre of Excellence, Nutrition and Non-Communicable Diseases, Faculty of Medicine and Health Sciences, Universiti Putra Malaysia, UPM, 43400 Serdang, Selangor, Malaysia. ${ }^{4}$ Department of Community Health, Faculty of Medicine \& Health Sciences, Universiti Putra Malaysia, UPM, 43400 Serdang, Selangor, Malaysia. ${ }^{5}$ Department of District Health Office, Batu Pahat District Health Office, 83000 Batu Pahat, Johor, Malaysia. 
Received: 23 June 2019 Accepted: 30 September 2019

Published online: 30 October 2019

\section{References}

1. World Health Organization. Report of the Commission on Ending Childhood Obesity: implementation plan: executive summary. 2016. https://www.who. int/end-childhood-obesity/final-report/en/. Accessed 16 Sept 2017.

2. World Health Organization. Joint Child Malnutrition estimates - Levels and trends (2018 edition). 2018. https://www.who.int/nutgrowthdb/estimates201 7/en/. Accessed 9 Jan 2019.

3. Poh BK, Ng BK, Haslinda MDS, Shanita SN, Wong JE, Budin SB, et al. Nutritional status and dietary intakes of children aged 6 months to 12 years: findings of the nutrition survey of Malaysian children (SEANUTS Malaysia). $\mathrm{Br}$ J Nutr. 2013;110(S3):S21-35.

4. Zalilah MS, Mirnalini K, Khor G, Merlin A, Bahaman A, Norimah K. Estimates and distribution of body mass index in a sample of Malaysian adolescents. Med J Malaysia. 2006;61(1):48-58.

5. Tee ES, Norimah AK, Hamid Jan JM, et al. Nutritional status of primary and secondary school children. In: MyBreakfast study of school children: findings, implications \& solutions symposium; 2015. p. 10-p.

6. Khambalia AZ, Lim SS, Gill T, Bulgiba AM. Prevalence and sociodemographic factors of malnutrition among children in Malaysia. Food Nutr Bull. 2012 33(1):31-42.

7. Institut of Public Health. National Health \& Morbidity Survey (NHMS) 2017 : Adolescent nutrition survey 2017. Malaysia: Institute for Public Health, National Institutes of Health, Ministry of Health Malaysia; 2017.

8. Hayashi C, Krasevec J, Kumapley R, Mehra V, de Onis M, Borghi E, et al. Levels and trends in child malnutrition. In: UNICEF/WHO/World Bank Group joint child malnutrition estimates: key findings of the 2017 edition; 2017.

9. Luder E, Alton I. The underweight adolescent. In: Guidelines for Adolescent Nutritional Services; 2005. p. 93-100.

10. Lazzeri G, Rossi F, Pammolli A, Pilato V, Pozzi T, Giacchi M. Underweight and overweight among children and adolescents in Tuscany (Italy). Prevalence and short-term trends. J Prev Med Hyg. 2008;49(1):13-21.

11. Halfon N, Larson K, Slusser W. Associations between obesity and comorbid mental health, developmental, and physical health conditions in a nationally representative sample of US children aged 10 to 17. Acad Pediatr. 2013;13(1):6-13.

12. Chung $\mathrm{K}-\mathrm{H}$, Chiou $\mathrm{H}-\mathrm{Y}, \mathrm{C}$, Ch $\mathrm{Y}-\mathrm{H}$. Psychological and physiological correlates of childhood obesity in Taiwan. Sci Rep. 2015;5:17439.

13. Morrison KM, Shin S, Tarnopolsky M, Taylor VH. Association of depression \& health related quality of life with body composition in children and youth with obesity. J Affect Disord. 2015;172:18-23.

14. Taylor VH, Forhan M, Vigod SN, Mclntyre RS, Morrison KM. The impact of obesity on quality of life. Best Pract Res CI En. 2013;27(2):139-46.

15. Smith E, Hay P, Campbell L, Trollor JN. A review of the association between obesity and cognitive function across the lifespan: implications for novel approaches to prevention and treatment. Obes Rev. 2011;12(9):740-55.

16. World Health Organization. Health in 2015: MDGs, millenium development goals to SDGs sustainable development goal. 2015. https://www.who.int/ gho/publications/mdgs-sdgs/en/. Accessed 16 Sept 2018

17. Ministry of Education M. Education System Chart. 2018. https://www.moe gov.my/index.php/en/arkib/sistem-pendidikan. Accessed 1 Oct 2017

18. Pedersen TP, Holstein BE, Flachs EM, Rasmussen M. Meal frequencies in early adolescence predict meal frequencies in late adolescence and early adulthood. BMC Public Health. 2013:13(1):445.

19. Perera T, Frei S, Frei B, Wong SS, Bobe G. Improving nutrition education in US elementary schools: challenges and opportunities. J Edu Pract. 2015; 6(30):41-50.

20. Lee ST, Wong JE, Shanita SN, Ismail MN, Deurenberg P, Poh BK. Daily physical activity and screen time, but not other sedentary activities, are associated with measures of obesity during childhood. Int J Environ Res Public Health. 2014;12(1):146-61.

21. Chong KH, Wu SK, Noor Hafizah Y, Bragt MC, Poh BK, Group SMS. Eating habits of Malaysian children: Findings of the South East Asian Nutrition Surveys (SEANUTS). Asia-Pac J Public Health. 2016;28(5_suppl):59S-73S.

22. Tee ES, Nurliyana AR, Norimah AK, Jan Mohamed HJB, Tan SY, Appukutty M, et al. Breakfast consumption among Malaysian primary and secondary school children and relationship with body weight status-Findings from the MyBreakfast Study. Asia Pac J Clin Nutr. 2018;27(2):421-32.
23. Nurul-Fadhilah A, Teo PS, Huybrechts I, Foo LH. Infrequent breakfast consumption is associated with higher body adiposity and abdominal obesity in Malaysian school-aged adolescents. PLoS One. 2013;8(3):e59297.

24. Poh BK, Rojroonwasinkul N, Le Nyugen BK, Budiman B, Ng LO, Soonthorndhada K, et al. Relationship between anthropometric indicators and cognitive performance in southeast Asian school-aged children. $\mathrm{Br} \mathrm{J}$ Nutr. 2013;110(S3):S57-64.

25. Nasir MTM, Norimah AK, Hazizi AS, Nurliyana AR, Loh SH, Suraya I. Child feeding practices, food habits, anthropometric indicators and cognitive performance among preschoolers in peninsular Malaysia. Appetite. 2012; 58(2):525-30.

26. Lee P, Cheah W, Chang C, Raudzah S. Childhood obesity, self-esteem and health-related quality of life among urban primary schools children in Kuching, Sarawak, Malaysia. Malays J Nutr. 2012;18(2):207-19.

27. Aday LA, Cornelius $\sqcup$. Designing and conducting health surveys: a comprehensive guide. 3rd ed. San Francisco: Jossey-Bass A Wiley Imprint; 2006

28. Yusop NBM, Shariff ZM, Hwu TT, Talib RA, Spurrier N. The effectiveness of a stage-based lifestyle modification intervention for obese children. BMC Public Health. 2018;18(1):299.

29. Bandura A. Health promotion from the perspective of social cognitive theory. Psychol Health. 1998:13(4):623-49.

30. Tee ES, Norimah AK, Zawiah H, Chin YS, Rasyedah AR. Effectiveness of a Nutrition Education Intervention for Primary School Children: The Healthy Kids Program, Malaysia; 2017.

31. Ministry of Health. Modul Latihan Katering Sihat. Nutrition Division Ministry of Health. 2018

32. Ministry of Education Malaysia. Panduan Pengurusan Kantin Sekolah Sihat 2011: Bahagian Pengurusan Sekolah Harian, Kementerian Pendidikan Malaysia, Putrajaya, Malaysia; 2011.

33. World Health Organization. WHO AnthroPlus for personal computers manual: software for assessing growth of the world's children and adolescents. 2009.

34. World Health Organization AnthroPlus solfware. https://www.who.int/ growthref/tools/en/. Accessed Oct 2016.

35. Williams DP, Going SB, Lohman TG, Harsha DW, Srinivasan SR, Webber LS, Berenson GS. Body fatness and risk for elevated blood pressure, total cholesterol, and serum lipoprotein ratios in children and adolescents. Am J Public Health. 1992:82(3):358-63.

36. Chin Y, Mohd NM. Eating behaviors among female adolescents in Kuantan district, Pahang, Malaysia. Pak J Nutr. 2009;8(4):425-32.

37. Kowalski KC, Crocker PR, Donen RM. The physical activity questionnaire for older children (PAQ-C) and adolescents (PAQ-A) manual. College of Kinesiology, University of Saskatchewan, Canada. 2004;87(1):1-38.

38. Aggio D, Fairclough S, Knowles Z, Graves L. Validity and reliability of a modified English version of the physical activity questionnaire for adolescents. Arch Public Health. 2016;74(1):3.

39. Chorpita BF, Yim L, Moffitt C, Umemoto LA, Francis SE. Assessment of symptoms of DSM-IV anxiety and depression in children: a revised child anxiety and depression scale. Behav Res Ther. 2000;38(8):835-55.

40. Sandín B, Chorot P, Valiente RM, Chorpita BF. Development of a 30-item version of the revised child anxiety and depression scale; 2010.

41. Raven J. Raven's e educational: Coloured progressive matrices (CPM). London: Pearson Assessment; 2008.

42. Varni JW, Seid M, Kurtin PS. PedsQL ${ }^{\text {TM }}$ 4.0: Reliability and validity of the Pediatric Quality of Life Inventory ${ }^{\mathrm{TM}}$ Version 4.0 Generic Core Scales in healthy and patient populations. Med Care. 2001;39:800-12.

43. Varni JW, Burwinkle TM, Seid M, Skarr D. The PedsQL ${ }^{\mathrm{TM} *} 4.0$ as a pediatric population health measure: feasibility, reliability, and validity. Ambul Pediatr. 2003;3(6):329-41.

44. Gupta SK. Intention-to-treat concept: a review. Perspect Clin Res. 2011;2(3):109.

45. Reimann C, Filzmoser P, Garrett RG, Dutter R. Statistical data analysis explained: applied environmental statistics with R: Wiley online library; 2008.

46. DeVault N, Kennedy T, Hermann J, Mwavita M, Rask P, Jaworsky A. It's all about kids: preventing overweight in elementary school children in Tulsa. J Am Diet Assoc. 2009;109(4):680-7.

47. Ruzita AT, MAB WA, Ismail M. The effectiveness of nutrition education programme for primary school children. Malays J Nutr. 2007;13(1):45-54

48. Shariff ZM, Bukhari SS, Othman N, Hashim N, Ismail M, Jamil Z, et al. Nutrition education intervention improves nutrition knowledge, attitude and practices of primary school children: a pilot study. Health Educ J. 2008; 11(1):119-32. 
49. Ishak SIZS, Chin YS, Taib MNM, Shariff ZM. School-based intervention to prevent overweight and disordered eating in secondary school Malaysian adolescents: a study protocol. BMC Public Health. 2016;16(1):1101.

50. Gunasekaran DD, Sharif R, Koon PB, Talib RA, Safii NS, Ahmad M. Juara Sihat ${ }^{\text {TM. }}$ Study Design of a School-based Childhood Obesity Nutrition Education Programme in Kuala Lumpur, Malaysia. Jurnal Sains Kesihatan Malaysia. 2018;16:119-27.

51. Ministry of Health Malaysia. Batu Pahat Health Centre: Monitoring food and beverages form KSS1/KKM; 2015.

52. Mensink F, Schwinghammer SA, Smeets A. The healthy school canteen programme: a promising intervention to make the school food environment healthier. J Environ Public Health. 2012;1-8.

53. Evenhuis I, Wezenbeek $\mathrm{N}$, Vyth E, Veldhuis L, Poelman M, Wolvers $\mathrm{D}$, et al. Development of the 'canteen scan': an online tool to monitor implementation of healthy canteen guidelines. BMC Public Health. 2018; 18(1):1109.

54. Micha R, Karageorgou D, Bakogianni I, Trichia E, Whitsel LP, Story M, et al. Effectiveness of school food environment policies on children's dietary behaviors: a systematic review and meta-analysis. PLoS One. 2018;13(3): e0194555.

55. Obbagy JE, Condrasky MD, Roe LS, Sharp JL, Rolls BJ. Chefs' opinions about reducing the calorie content of menu items in restaurants. Obesity. 2011; 19(2):332-7.

56. Lessa K, Cortes C, Frigola A, Esteve M. Food healthy knowledge, attitudes and practices: survey of the general public and food handlers. Int J Fastron Food Sci. 2017;7:1-4

57. Tanaka N, Miyoshi M. School lunch program for health promotion among children in Japan. Asia Pac J Clin Nutr. 2012;21(1):155-8.

58. Daniels SR, Hassink SG. The role of the pediatrician in primary prevention of obesity. Pediatrics. 2015;136:e275-92.

59. Koo HC, Poh BK, Abd TR. The GReat-childTm trial: a quasi-experimental intervention on whole grains with healthy balanced diet to manage childhood obesity in Kuala Lumpur, Malaysia. Nutrients. 2018;10(2):156.

60. Miyoshi M, Tsuboyama-Kasaoka N, Nishi N. School-based "Shokuiku" program in Japan: application to nutrition education in Asian countries. Asia Pac J Clin Nutr. 2012;21(1):159-62.

61. Ministry of Health Malaysia. National Plan of Action for Nutrition of Malaysia [NPANM] III (2016-2025). 1st ed: National Coordinating Committee on Food and Nutrition (NCCFN), Putrajaya, Malaysia; 2015.

\section{Publisher's Note}

Springer Nature remains neutral with regard to jurisdictional claims in published maps and institutional affiliations.

Ready to submit your research? Choose BMC and benefit from:

- fast, convenient online submission

- thorough peer review by experienced researchers in your field

- rapid publication on acceptance

- support for research data, including large and complex data types

- gold Open Access which fosters wider collaboration and increased citations

- maximum visibility for your research: over $100 \mathrm{M}$ website views per year

At $\mathrm{BMC}$, research is always in progress.

Learn more biomedcentral.com/submissions 